\title{
On the Confinement of Passing Alpha Particles in a Tokamak-Reactor with Resonant Magnetic Field Perturbations Shielded by Plasma Currents
}

\author{
M. F. Heyn ${ }^{1}$, I. B. Ivanov ${ }^{1,2}$, S. V. Kasilov ${ }^{1,3}$ W. Kernbichler ${ }^{1}$, \\ A. Loarte $^{4}$, V. V. Nemov ${ }^{1,3}$, and A. M. Runov ${ }^{5}$ \\ ${ }^{1}$ Institut für Theoretische Physik - Computational Physics, Technische \\ Universität Graz, Association EURATOM-OEAW, A-8010 Graz, Austria \\ ${ }^{2}$ Petersburg Nuclear Physics Institute, 188300, Gatchina, Leningrad Region, Russia \\ ${ }^{3}$ Institute of Plasma Physics, National Science Center "Kharkov \\ Institute of Physics and Technology", 61108 Kharkov, Ukraine \\ ${ }^{4}$ ITER Organization, Route de Vinon sur Verdon, 13115 St Paul lez Durance, France \\ ${ }^{5}$ Max-Planck Institut für Plasmaphysik, EURATOM \\ Association, D-17491 Greifswald, Germany
}

\begin{abstract}
Alpha-particle losses due to the resonant magnetic field perturbations (RMPs) created by the coil system for Edge Localized Mode mitigation in ITER are studied numerically. If shielding of RMPs by the plasma is not taken into account, passing $\alpha$-particles are the main loss channel which, together with the trapped particle contribution leads to a loss of more than $5 \%$ of fusion alpha particle power. Shielding of RMPs practically eliminates this channel so that the overall losses are reduced to about $1 \%$.
\end{abstract}




\section{INTRODUCTION}

Resonant magnetic field perturbations (RMPs) produced with a special coil set can be successfully used for mitigation of Edge Localized Modes (ELM'S) in tokamaks what has been demonstrated experimentally on DIII-D ${ }^{1}$ and JET $^{2}$. They are considered as an ELM mitigation tool in $\mathrm{ITER}^{3}$. If such RMPs are considered ignoring the effects of the plasma ("vacuum" RMPs), they can cause ergodization of the magnetic field in a significant part of the plasma volume, which should result in a noticeable reduction of the energy confinement time ${ }^{4}$. Such strong ergodization should also have a negative effect on the confinement of fast passing particles, in particular, of fusion $\alpha$-particles. However, the reduction of energy confinement time predicted from the vacuum RMP model is not observed experimentally ${ }^{4}$. One of the most likely reasons is that the perturbations are strongly shielded by plasma response currents at resonant rational magnetic surfaces ${ }^{5}$. Predictions of such shielding by linear kinetic ${ }^{5}$ and drift $\mathrm{MHD}^{6}$ models agree by order of magnitude. As a result of the shielding, the island width which follows from the vacuum model for the perturbation field is reduced up to two orders of magnitude. This reduction comes from the strong drop of the radial component of the perturbation field in a narrow region around the resonant surface where this component has a minimum. Due to their cross-field drift, passing $\alpha$-particles do not stay on magnetic surface, and, therefore, for resonant orbits, they may experience noticeably higher values of the radial magnetic field than its value at the rational magnetic surface. Therefore, the topology of such orbits is more sensitive to RMPs than the topology of field lines.

In the following, orbit topology and particle losses are studied for the 15 MA ITER QDT

$=10$ scenario with perturbation coil system ${ }^{7-9}$ with the help of the magnetic field model used in Ref. 5. This model has been used earlier for the estimation of RMP effects on the magnetic configuration in the presence of a plasma typical for experiments on ELM mitigation in DIII-D. In this model, the realistic axisymmetric equilibrium magnetic field for the $2 \mathrm{D}$ tokamak geometry and the realistic $3 \mathrm{D}$ vacuum RMP field is coupled with the $1 \mathrm{D}$ linear kinetic model of RMP shielding in screw pinch geometry. The resulting 3D shielded perturbation field is added to the 2D equilibrium field and the total field is used for the computation of the orbits. This model is described in Section II. In Section III the topology of field lines and passing $\alpha$-particle orbits for the vacuum RMP model and for shielded 
RMP model is studied with help of Poincaré mapping. In Section IV passing and trapped $\alpha$-particle losses are computed. Results are summarized in Section V.

\section{MAGNETIC FIELD MODEL}

The magnetic field is given as a superposition of the unperturbed field and the perturbation field, $\mathbf{B}=\mathbf{B}_{0}+\tilde{\mathbf{B}}$ where the unperturbed (axisymmetric) magnetic field is given by

$$
\mathbf{B}_{0}=\nabla A_{0 \varphi} \times \nabla \varphi+B_{0 \varphi} \nabla \varphi
$$

Here, $B_{0 \varphi}$ is the co-variant toroidal magnetic field component (constant in the low pressure approximation) and $A_{0 \varphi}=-\psi_{\mathrm{pol}}$ is the co-variant toroidal component of the vector potential which are the standard output of tokamak equilibrium codes.

In absence of a plasma, the perturbation field $\tilde{\mathbf{B}}$ is fully determined by the currents in the perturbation coil system via the Biot-Savart law. In the present study, the modelling is done for the coil system and coil currents taken from Refs. 7,8. Such coil system for ITER consists of 9 toroidal sectors containing 3 coils each (see Fig. 1) denoted as upper (u), equatorial (e) and lower (l). The particular mode considered here is characterized by the following currents in these coils,

$$
I_{s}^{(c)}=I_{A} \sin \left(n_{m}(s \Delta \varphi-\Delta \varphi(c))\right)
$$

where $s=1, \ldots, 9$ is the sector number, $(c)=(u),(e),(l)$ is the coil type, the amplitude of the current is $I_{A}=90 \mathrm{kA}$, the sector period is $\Delta \varphi=40^{\circ}$, and phase shifts for various coil types are $\Delta \varphi_{(u)}=54^{\circ}, \Delta \varphi_{(e)}=0^{\circ}$ and $\Delta \varphi_{(l)}=-64^{\circ}$. For the perturbation mode $n_{m}=4$ the toroidal spectrum of the current essentially consists of the modes with $n=4+9 k$ and $n=5+9 k$ where $k$ is an integer. Other modes with much smaller amplitudes are also present in the spectrum because the discrete symmetry of the sectors is violated by the different current feeds of the lower coil in sector 2. Although this violation is small, periodicity of the coils with period number 9 has not been assumed in the present computations. 


\section{A. Vacuum perturbation field}

For a fast numerical evaluation, the perturbation field $\tilde{\mathbf{B}}$ computed with the Biot-Savart law is represented in cylindrical coordinates $(R, \varphi, Z)$ through vector-potential components,

$$
\tilde{\mathbf{B}}=\nabla \tilde{A}_{R} \times \nabla R+\nabla \tilde{A}_{Z} \times \nabla Z+\nabla \bar{A}_{\varphi} \times \nabla \varphi+\bar{B}_{\varphi} \nabla \varphi
$$

where $\bar{A}_{\varphi}$ and $\bar{B}_{\varphi}$ are independent of the toroidal angle $\varphi$ and the components $\tilde{A}_{R}$ and $\tilde{A}_{Z}$ have zero toroidal average. These components are expanded in Fourier series over $\varphi$, and the coefficients of this series are used in the form of $2 \mathrm{D}$ spline interpolation of the pre-computed data on the $(R, Z)$ grid. Such a representation is divergence-free up to computer accuracy and, in addition, is helpful for the analysis of the perturbation field in flux coordinates. In symmetry flux coordinates $\left(\psi_{\text {tor }}, \vartheta, \varphi\right)$ being the toroidal flux, poloidal and toroidal angles, respectively, which are constructed numerically from the equilibrium field data, the nonaxisymmetric part of the perturbation field takes the form

$$
\tilde{\mathbf{B}}=\nabla \tilde{A}_{\psi_{\text {tor }}} \times \nabla \psi_{\text {tor }}+\nabla \tilde{A}_{\vartheta} \times \nabla \vartheta
$$

The component $\tilde{A}_{\psi_{\text {tor }}}$ does not modify the magnetic field topology because this small term can be iteratively absorbed into the flux coordinates. In turn, the component $\tilde{A}_{\vartheta}$ determines both the topology of the total field and the resonant response currents. Representing the components of the perturbed vector potential in the form of a Fourier series,

$$
\tilde{A}_{\vartheta}=2 \operatorname{Re} \sum_{n=1}^{\infty} \sum_{m=-\infty}^{\infty} A_{\vartheta ; m, n}\left(\psi_{\text {tor }}\right) \mathrm{e}^{i m \vartheta+i n \varphi}
$$

the Fourier amplitudes $A_{\vartheta ; m, n}$ and $A_{\psi_{\text {tor } ; m, n}}$ in the absence of plasma response currents are computed by numerical integration of $\tilde{A}_{R}$ and $\tilde{A}_{Z}$ along the unperturbed field lines ${ }^{5}$.

\section{B. Account of shielding}

To account of plasma response currents, the Fourier amplitudes are expressed through the amplitudes in vacuum and the "formfactors" $T_{m, n}$ as follows,

$$
A_{\vartheta ; m, n}^{(\mathrm{plas})}\left(\psi_{\text {tor }}\right)=A_{\vartheta ; m, n}^{(\mathrm{vac})}\left(\psi_{\text {tor }}\right) T_{m, n}\left(\psi_{\text {tor }}\right)
$$

For the estimation of the formfactors in the presence of plasma response, the model of an inhomogeneous periodic plasma cylinder with rotational transform (screw pinch) is used. In 
this estimate, the formfactors for various Fourier modes are set to the respective formfactors in the screw pinch tokamak model,

$$
T_{m, n}\left(\psi_{\text {tor }}\right)=B_{r ; m, n}^{(p l a s)}(r) / B_{r ; m, n}^{(v a c)}(r)
$$

where $r=\sqrt{2 \psi_{\text {tor }} / B_{\text {axis }}}$ is the effective radius and $B_{\text {axis }}$ is a reference magnetic field. The Fourier amplitudes of the magnetic field components in the cylinder, $B_{r ; m, n}$, are computed numerically using the linear kinetic model introduced in Ref. 10 and recently upgraded in Ref. 11. Within this model, Maxwell's equations,

$$
\nabla \times \tilde{\mathbf{E}}=\frac{i \omega}{c} \tilde{\mathbf{B}}, \quad \nabla \times \tilde{\mathbf{B}}=-\frac{i \omega}{c} \tilde{\mathbf{E}}+\frac{4 \pi}{c} \tilde{\mathbf{j}}
$$

are solved for a cylindrical plasma surrounded by an ideally conducting wall at $r=r_{W}$. Perturbations are excited by the "antenna" at $r=r_{A}<r_{W}$. The position of the antenna has no effect on $T_{m, n}$ as long as the plasma density and the equilibrium plasma current at the antenna are negligibly small. The same is true for the wall position if the wall is distant enough from the plasma,

$$
\left(\frac{r_{p}}{r_{W}}\right)^{2(|m|-1)} \ll 1
$$

where $r_{p}$ is radius of the separatrix. For quasi-static perturbations, the conductivity of the wall is negligibly small and formally one has to place the ideally conducting wall at infinity. However, poloidal modes $m$ which are resonant in the plasma are large for the coil spectra of interest here, such that condition (9) is well satisfied even with rather moderate $r_{W}$ values. The plasma response current density in (8) is computed from

$$
\tilde{\mathbf{j}}=\sum_{e, i} e \int \mathrm{d}^{3} p \mathbf{v} \tilde{f}
$$

where the perturbed distribution function $\tilde{f}$ satisfies the linearized kinetic equation

$$
\frac{\partial \tilde{f}}{\partial t}+\mathbf{v} \cdot \nabla \tilde{f}+e\left(-\nabla \Phi_{0}+\frac{1}{c} \mathbf{v} \times \mathbf{B}_{0}\right) \cdot \frac{\partial \tilde{f}}{\partial \mathbf{p}}-\hat{L}_{C} \tilde{f}=-e\left(\tilde{\mathbf{E}}+\frac{1}{c} \mathbf{v} \times \tilde{\mathbf{B}}\right) \cdot \frac{\partial f_{0}}{\partial \mathbf{p}} .
$$

Here $f_{0}$ is an equilibrium distribution function consistent with plasma and equilibrium magnetic field parameter profiles, taking toroidal plasma rotation into account and assuming that poloidal rotation velocity is zero, $\Phi_{0}$ is the equilibrium electrostatic potential and $\hat{L}_{C}$ models Coulomb collisions. In more detail, the equilibrium is specified by the radial profiles of density $n_{e}$, electron temperature $T_{e}$, ion temperature $T_{i}$, toroidal rotation velocity $V_{z}$, 
safety factor $q$, and by the on-axis magnetic field value $B_{0}(0)$. The parallel plasma current, $j_{\|}=|e| n_{e}\left(V_{\|, i}-V_{\|, e}\right)$, and the components of the equilibrium magnetic field $B_{0}^{\theta}$ and $B_{0}^{z}$ follow then from $q(r)$ and $B_{0}(0)$ via Ampére's law. The perpendicular electron and ion fluid velocities $\mathbf{V}_{\perp e, i}=\mathbf{V}_{* e, i}+\mathbf{V}_{E}$, with $\mathbf{V}_{* e, i}$ the diamagnetic velocities and $\mathbf{V}_{E}$ the electric drift velocity, are determined by the ideal MHD force balance ignoring inertia. Then the poloidal and toroidal projections of the total fluid velocity, $m_{e} \mathbf{V}_{\perp e}+m_{i} \mathbf{V}_{\perp i}$, together with the definition of the parallel current density provide an equation set for the electric field and the parallel fluid velocity components. These parameters fully determine the equilibrium distribution function in form of a shifted Maxwellian with radial dependence.

A schematic model showing the origin of the shielding can be obtained for a single static perturbation field harmonic, $\tilde{\mathbf{B}} \propto \exp \left(i m_{\vartheta} \vartheta+i k_{z} z\right)$, ignoring the perturbed electrostatic potential and using a gyrokinetic equation with Krook collision term,

$$
\mathbf{V}_{g} \cdot \nabla f+\nu_{c}\left(f-f_{0}\right)=0
$$

where the velocity space variables are the total energy $w$ and the perpendicular adiabatic invariant $J_{\perp}$. In a common transport ansatz, the zero order distribution function is a local Maxwellian,

$$
f_{0}=\frac{n(r)}{(2 \pi m T(r))^{3 / 2}} \exp \left(-\frac{w-e \Phi_{0}(r)}{T(r)}\right) .
$$

Ignoring the magnetic drift in the guiding center velocity,

$$
\mathbf{V}_{g}=v_{\|} \frac{\mathbf{B}}{B}+\mathbf{V}_{E}
$$

linearization of (12) with respect to $\tilde{\mathbf{B}}$ leads to

$$
i\left(k_{\|} v_{\|}+\omega_{E}-i \nu_{c}\right) \tilde{f}=-v_{\|} \frac{\tilde{B}^{r}}{B_{0}} \frac{\partial f_{0}}{\partial r}, \quad \frac{\partial f_{0}}{\partial r}=\left(A_{1}+A_{2} \frac{w-e \Phi_{0}}{T}\right) f_{0}
$$

where $k_{\|}=\mathbf{B}_{0} \cdot \mathbf{k} / B_{0}, \omega_{E}=\mathbf{V}_{E} \cdot \mathbf{k}, \mathbf{k}=m_{\vartheta} \nabla \vartheta+k_{z} \nabla z$, and the thermodynamic forces are

$$
A_{1}=\frac{1}{n} \frac{\partial n}{\partial r}+\frac{e}{T} \frac{\partial \Phi_{0}}{\partial r}-\frac{3}{2 T} \frac{\partial T}{\partial r}, \quad A_{2}=\frac{1}{T} \frac{\partial T}{\partial r} .
$$

Substituting the solution to (15) into (10), the parallel perturbation current is obtained as

$$
\tilde{j}_{\|}=\sigma_{B} \tilde{B}^{r}=\sum_{e, i} \frac{e n T \tilde{B}^{r}}{m B_{0}\left(\nu_{c}+i \omega_{E}\right)} \frac{2 Z}{\sqrt{\pi}} \int_{-\infty}^{\infty} \frac{\mathrm{d} u u^{2}}{u-Z} \mathrm{e}^{-u^{2}}\left(A_{1}+A_{2}+A_{2} u^{2}\right)
$$


with $Z=\left(i \nu_{c}-\omega_{E}\right) k_{\|}^{-1}(2 T / m)^{-1 / 2}$. This current is peaked at the resonant surface where $k_{\|}=0$ and $Z \rightarrow \infty$,

$$
\tilde{j}_{\|}=-\sum_{e, i} \frac{e \tilde{B}^{r}}{m B_{0}\left(\nu_{c}+i \omega_{E}\right)}\left(\frac{\partial n T}{\partial r}+e n \frac{\partial \Phi_{0}}{\partial r}\right)=-\sum_{e, i} \frac{e^{2} n \tilde{B}^{r} V_{\perp}}{m c\left(\nu_{c}+i \omega_{E}\right)},
$$

i.e., the current is driven by the magnetic Lorentz force arising from the perturbation field and the moving fluid, a result which is also seen in MHD theory. ${ }^{12}$ Here and below subscript $\perp$ refers to the perpendicular component of the vector within the flux surface. Due to the mass ratio, the electron current is much larger then the ion current, especially in the hot core where $\omega_{E}$ may be higher than the electron collision frequency. Away from the resonant surface where $Z$ becomes small due to increasing $k_{\|}$, the current is decreasing

$$
\tilde{j}_{\|} \approx \frac{e \tilde{B}^{r}\left(i \nu_{c}-\omega_{E}\right)}{B_{0} k_{\|}^{2}}\left(\frac{\partial n}{\partial r}+\frac{e n}{T} \frac{\partial \Phi_{0}}{\partial r}\right)
$$

The width of the current layer where $Z \geq 1$ is rather small. E.g., for $\omega_{E}>\nu_{c}$ this width is

$$
\Delta r \sim \frac{c k_{\perp} q}{B_{0} k_{z}} \sqrt{\frac{m}{T}} \frac{\partial \Phi_{0}}{\partial r}\left(\frac{\partial q}{\partial r}\right)^{-1} \sim \frac{\rho q R}{r}
$$

where $\rho$ is the Larmor radius and $R$ is the major radius of the torus. The current in this layer shields the perturbation field, and the main shielding results from the electrons because the total current of a given species contained in the layer between the resonant surface and the coil scales inversely with the square root of mass. This immediately follows from the estimate of the current amplitude (18) and its width (20).

Generally, the current density (10) is an integral functional of the perturbation field and, due to finite Larmor radius (FLR) effect, it remains to be such even in cylindrical geometry where Fourier analysis over poloidal and toroidal angles transforms the integral nonlocality over these angles into an algebraic dependence. In Ref. 10 a particular FLR expansion has been introduced which preserves the Galilean covariance of the resonant part of current density (part responsible for the shielding effect) such that the integral plasma conductivity operator is approximated by a differential operator. This allows to reduce Maxwell equations to a set of coupled ordinary differential equations. Using Galilean covariance, Maxwell equations are solved in some moving (toroidally rotating) reference frame where the perturbation frequency $\omega$ is finite. Originally, this model had been realized for the lowest order expansion only ${ }^{10}$. In Ref. 11, more practical modified higher order 
expansions have been developed and implemented numerically. In addition, the Krook collision operator used in Refs. 5,10 for the estimation of Coulomb collision effects on the shielding has been replaced with a simple Fokker-Planck type collision operator, namely an Ornstein-Uhlenbeck operator ${ }^{13}$. This operator conserves particles locally and, as a result, does not violate Galilean covariance of the current in contrast to the Krook operator used for estimates in Refs. 5,10 where this covariance has been held only in the collisionless case. It should be mentioned that poloidal mode coupling pertinent to the realistic 2D toroidal geometry is ignored in the cylindrical model used here. This coupling provides corrections which are relatively small in the core plasma being of the main interest for $\alpha$-particle confinement.

For numerical computations, plasma parameters and equilibrium magnetic field for the 15 MA ITER QDT $=10$ scenario $^{9}$ are used. Profiles of these parameters as functions of the normalized poloidal flux $s=\psi_{\text {pol }} / \psi_{\text {pol }}^{a}$ being the poloidal flux normalized by its value at the separatrix, are shown in Fig. 2. The resulting formfactors in Fig. 3 show strong shielding of the perturbation field in the plasma.

\section{FIELD LINES AND DRIFT ORBITS OF PASSING $\alpha$-PARTICLES}

The results of field line tracing with and without the account of RMP shielding by the plasma are presented in Fig. 4 where Poincaré plots at the $\varphi=0$ section are shown in flux variables $(s, \vartheta)$. It can be seen that large magnetic islands in the core plasma and a strong ergodization in the outer two thirds of the plasma volume are almost eliminated if RMP shielding by the plasma is taken into account. The reduction of the island widths in the core is up to 100 times because the values of the formfactors at the respective resonant surfaces are down to $10^{-4}$ there, see Fig. 3, and the island width scales as the square root of the perturbation field amplitude. At the same time, it can be seen from Fig. 3 that such strong drops in the formfactors occur in rather narrow vicinities of the resonant flux surfaces. The typical radial scale of such a vicinity is comparable to the Larmor radius of thermal ions. Since the deviation of $\alpha$-particles from the magnetic surfaces is much larger than this scale, one can expect that the effect of RMPs on $\alpha$-particle orbits is much stronger than the effect on magnetic field lines. In addition, Larmor gyration of $\alpha$-particles also reduces the effect of plasma shielding of the RMP field for the orbits. This relatively small effect, however, is not 
considered below where the $\alpha$-particle motion is modelled in the usual drift approximation. Drift orbits of passing $\alpha$-particles can be presented as the lines of force of the effective magnetic field $\mathbf{B}^{*}$ (see Ref. 14) where

$$
\mathbf{B}^{*}=\nabla \times \mathbf{A}^{*}, \quad \mathbf{A}^{*}=\mathbf{A}+\frac{m_{\alpha} c v_{\|}}{e_{\alpha} B} \mathbf{B} \equiv \mathbf{A}+\frac{v_{\|}}{\omega_{c \alpha}} \mathbf{B} .
$$

Here, $e_{\alpha}, m_{\alpha}$ and $\omega_{c \alpha}$ are $\alpha$-particle charge, mass and cyclotron frequency, respectively. Parallel velocity $v_{\|}$in (21) must be expressed in terms of invariants of motion being the total energy $w$ and perpendicular invariant $J_{\perp}=p_{\perp}^{2}\left(2 m_{\alpha} \omega_{c \alpha}\right)^{-1}$ as follows

$$
v_{\|}=\sigma \sqrt{\frac{2}{m_{\alpha}}\left(w-\omega_{c \alpha} J_{\perp}-e_{\alpha} \Phi_{0}\right)},
$$

where $\sigma= \pm 1$ is the velocity sign. For the analysis of orbit topology, Poincaré mapping of the effective magnetic field (21) has been performed for strongly passing particles with $J_{\perp}=0$ ignoring the electrostatic potential $\Phi_{0}$ which is important for bulk plasma particles but provides a negligible correction for high energy $\alpha$-particles. In this case, $v_{\|}= \pm v_{0}$ where $v_{0}$ is the velocity of $3.5 \mathrm{MeV} \alpha$-particle. For simplicity, the perturbation field has been taken into account only for the first term in the right hand side of (21). The neglected small term could be important for trapped particles since it describes toroidal field ripple due to the perturbation field. However, it provides just a minor correction for passing particles, and this approximation does not lead to non-Hamiltonian features of the orbits.

The results of Poincaré mapping are shown in Fig. 5. In all cases there is a visible difference between Poincaré plots for co- and counter-passing particles which is pre-determined by the difference in the unperturbed orbits which is induced by the radial drift in the toroidal field (see lower plots in Fig. 5). Such a difference has been demonstrated earlier in Ref. 15 for the vacuum perturbation field of TEXTOR-DED. As expected, the effect of shielding on particle orbits is weaker than on field lines. Relatively large drift islands corresponding to low order resonances can be observed for counter-passing orbits in the presence of the shielding by the plasma. Nevertheless, this shielding is sufficient to limit the size of the region occupied by ergodic orbits significantly. Moreover, islands are practically absent for the co-passing orbits.

To study the reduction of the shielding effect for the orbits it is useful to introduce symmetry flux coordinates $\psi_{\text {tor }}^{*}$ and $\vartheta^{*}$ associated with the effective magnetic field $\mathbf{B}_{0}^{*}$. In these coordinates, the spectrum of the perturbation field produced by a single harmonic $(m, n)$ 
in usual flux coordinates (which produces a single magnetic island chain) is determined by Fourier expansion over $\vartheta^{*}$ of the poloidal vector potential component $A_{\vartheta, m n}\left(\psi_{\text {tor }}\right) \exp (i m \vartheta)$ with substitution $\psi_{\text {tor }}=\psi_{\text {tor }}^{*}+\Delta \psi\left(\psi_{\text {tor }}^{*}, \vartheta^{*}\right)$ and $\vartheta^{*} \approx \vartheta$. Here we ignored the difference between $\vartheta^{*}$ and $\vartheta$ which is the same order correction with the banana width correction $\Delta \psi$ because of the stronger variation of the perturbation field with $\psi_{\text {tor }}$ near the resonance. Thus, besides the increase of the amplitude of the main harmonic $(m, n)$ discussed above and illustrated in Fig. 6 there appear also sideband harmonics due to the coupling of the periodic function $\Delta \psi$ with the exponent through the dependence of $A_{\vartheta, m n}\left(\psi_{\text {tor }}\right)$ on the argument. The largest sidebands correspond to $m \pm 1$. In Poincaré plots of passing orbits with the single perturbation harmonic $m=6, n=-4$ (upper plots in Fig. 7) one can see both, the island chain due to the main harmonic and due to the sideband $m+1=7$ whose resonant surface is located outside the main resonance surface. The other nearest sideband $m-1=5$ located inside is practically absent because of shielding. Note that the main island chain for the counter passing orbit is visibly smaller than for co-passing since deviation of this orbit to the outer side of the main resonant surface where the perturbation field is strong is smaller (see Fig. 6). A similar behavior is seen also for the mode $m=5, n=-4$ (middle plots in Fig. 7). It is remarkable that for superposition of these two modes (lower plots in Fig. 7) the island chain $m=6, n=-4$ nearly vanishes for the co-passing orbit and is amplified for the counter-passing because in the first case the main resonant harmonic for the $m=6, n=-4$ perturbation mode is nearly cancelled by the sideband $m+1$ for the $m=5, n=-4$ mode while in the second case the sign of the sideband is opposite, and this harmonic is amplified. This feature results in very different topology of co- and counter-passing orbits in the core plasma for the full coil spectrum (middle plots of Fig. 5).

\section{PARTICLE AND ENERGY LOSSES}

For the quantitative evaluation of $\alpha$-particle losses, particles are split into three classes as follows. Note that the maximum value of the magnetic field seen by the passing particle during a poloidal turn is increasing when it travels outwards. Therefore, the part of the particles which are passing in the usual sense will be trapped on their way out. Introducing the normalized perpendicular invariant, $\eta=J_{\perp} e_{\alpha} B_{\max }^{(\mathrm{sep})}\left(m_{\alpha} c w\right)^{-1}$, where $B_{\max }^{(\mathrm{sep})}$ is the maximum value of the magnetic field at the unperturbed separatrix, "passing" particles are 
re-defined as those which can reach the separatrix without trapping, i.e. as particles with $\eta<1$. Particles with $\eta>B_{\max }^{(\mathrm{sep})} / B_{\max }^{(\mathrm{brt})}$ which are trapped on their birth surface, where $B_{\max }^{(\text {brt }}$ is the maximum value of the magnetic field at this surface are called below "trapped", and particles with $1<\eta<B_{\max }^{(\text {sep })} / B_{\max }^{(\text {brt })}$ which are trapped on the way to the separatrix are called "transient".

\section{A. Passing particles}

The standard procedure for the evaluation of $\alpha$-particle losses for realistic 3D magnetic fields ${ }^{16}$ assumes numerical tracing of drift orbits for a relatively large ensemble of particles representative for all $\alpha$-particles in the device. These particles must be followed for a time comparable with the $\alpha$-particle slowing down time by electrons which is of the order of one second in ITER (see Fig. 8). Naturally, this results in rather large CPU time requirements. To speed up the computations of passing particle losses, in the present study an interpolated cell mapping procedure ${ }^{17-19}$ has been applied which had been successfully used earlier for the modelling of run-away electron confinement ${ }^{17}$, 3D MHD transport modelling of the plasma edge ${ }^{18}$, and long mean free path transport modelling in stellarators ${ }^{19}$. Within this procedure, $\alpha$-particles are traced by their footprints on Poincaré cuts which are the poloidal cuts $\varphi=$ const cutting the torus in each field period. Cylindrical coordinates $(R, Z)$ of the particles on the cut are subsequently mapped through a period as follows,

$$
R_{\text {new }}=\mathcal{R}\left(R_{\text {old }}, Z_{\text {old }}\right), \quad Z_{\text {new }}=\mathcal{Z}\left(R_{\text {old }}, Z_{\text {old }}\right),
$$

where the mapping functions $\mathcal{R}(R, Z)$ and $\mathcal{Z}(R, Z)$ are used in the form of interpolation of their values pre-computed on the rectangular grid of starting values $(R, Z)$ by direct orbit integration over a field period. In addition to these functions also the single return time to the Poincaré cut, $\tau_{b}(R, Z)$, and the normalized slowing down rate $r_{s}(R, Z)=\tau_{b} / \tau_{s}$ are interpolated (here $\tau_{s}=\tau_{s}(R, Z)$ is the $\alpha$-particle slowing down time by electrons) and are summed up during the mappings. These sums determine the life time of the test particle, $\sum \tau_{b}$, and the fraction of the initial particle energy carried away by the particle, $\exp \left(-2 \sum r_{s}\right)$. Since in the presence of RMP shielding by the plasma the magnetic field has a rather steep behavior with respect to the flux surface label near the resonant flux surfaces, in the present work 5-th order 2D Lagrange polynomials are used for interpolation, in contrast to Refs. 17-19 
where bi-cubic splines have been used. This reduces the memory requirement by a factor 16 as compared to bi-cubic splines for the cost of some not very significant slowing down of the computations. Such a procedure allows to follow $10^{5}$ orbits for several million full turns around the torus what is sufficient to cover a few slowing down times. Therefore, the ergodic region at the edge can be resolved in sufficient detail. In order to cover the whole passing particle population separate mappings have been performed for a set of $\eta$ values.

In Fig. 9 are shown as functions of time after birth the lost fraction of particles born with trapping parameter $\eta$ at the flux surface with label $s, p_{L}^{(p)}=p_{L}^{(p)}(\eta, s)$ and relevant fraction of fusion energy $p_{L}^{(e)}=p_{L}^{(e)}(\eta, s)$ lost together with these particles. Plots correspond to two different flux surfaces and strongly counter-passing particles $(\eta=0)$. These fractions have the meaning of loss probability averaged over the volume between two neighboring flux surfaces with the weight equal to the Jacobian of the momentum space variables $J \propto B\left|v_{\|}\right|^{-1}$. Difference between $p_{L}^{(p)}$ and $p_{L}^{(e)}$ which develops for times $t$ comparable or larger than slowing down time is due to the energy transfer to bulk plasma electrons before particle loss. This difference is rather small for the vacuum perturbation field where particles are lost promptly almost with their initial energy, but it is large for the shielded field where particles diffuse to the wall for a long time sufficient for thermalisation of the major part of their energy. In Fig. 10 the dynamics of losses is shown for particles with $\eta=0$ with positive and negative parallel velocity. For several time moments, the fractions of particles $p_{L}^{(p)}=p_{L}^{(p)}(\eta, s)$ and energy $p_{L}^{(e)}=p_{L}^{(e)}(\eta, s)$ lost to the wall are plotted as functions of the starting value of the surface label $s$. For reference, also losses of "field lines" i.e. of the orbits in infinitely strong magnetic field are presented. It can be seen that losses in vacuum (left panel) are rather prompt everywhere: quantities $p_{L}^{(p)}$ and $p_{L}^{(e)}$ are close to each other, i.e. practically all $\alpha$-particles born at $s>0.6$ are lost to the wall almost with their initial energy. Note that dips on the $p_{L}$ profiles correspond to the residual islands in the ergodic layer where a small part of the alphas is confined (compare to Figs. 4 and 5). In the plasma, the loss region is significantly reduced by shielding. This reduction is strongest for "field lines" and is different for co- and counter-passing particles. 


\section{B. Trapped particles}

Trapped particle losses have been computed using the usual method ${ }^{16}$, i.e. particles have been started on several unperturbed flux surfaces evenly distributed over the volume between two neighboring surfaces and with isotropic momentum space distribution in the trapped particle domain and followed during $100 \mathrm{~ms}$. These losses have been estimated only for the vacuum perturbation case. In Fig. 11 is shown the dynamics of particle loss probability averaged over trapped particle domain,

$$
\left\langle p_{L}^{(p)}\right\rangle_{t r}=\frac{1}{\eta_{\max }-\eta_{\min }} \int_{\eta_{\min }}^{\eta_{\max }} \mathrm{d} \eta p_{L}^{(p)}(\eta, s)
$$

where $\eta_{\min }=B_{\max }^{(\mathrm{sep})}\left(B_{\max }^{(\mathrm{brt})}\right)^{-1}, \eta_{\max }=B_{\max }^{(\mathrm{sep})}\left(B_{\min }^{(\mathrm{brt})}\right)^{-1}$, with $B_{\min }^{(\mathrm{brt})}$ being the minimum magnetic field value at the birth surface. This losses are also prompt, i.e. main losses occur within first $10 \mathrm{~ms}$ where the slowing down is not significant (therefore $\left\langle p_{L}^{(e)}\right\rangle_{t r}$ has been set to $\left\langle p_{L}^{(p)}\right\rangle_{t r}$ for trapped particles in the following). At the same time, the loss probability in the core plasma is significantly smaller than the equivalent probability for passing particles in vacuum. In Fig. 12 the distribution of $p_{L}^{(p)}(\eta, s)$ is presented. For convenience, the trapping parameter $\kappa=\left(\eta-\eta_{\min }\right)\left(\eta_{\max }-\eta_{\min }\right)^{-1}$ is used instead of $\eta$. It can be seen that losses are localized mainly near the usual trapped-passing boundary, $\kappa=0$, where the overlapping of drift-orbit resonances (resonances between poloidal bounce frequency $\omega_{b}$ and toroidal rotation frequency $\omega_{t}$ ) takes place leading to stochastic ripple diffusion ${ }^{20}$. These losses have the same nature as the losses of fast particles due to the toroidal field ripple. Since they are not sensitive to the magnetic field topology, shielding effects are not expected to reduce these losses significantly.

\section{Total losses}

Total losses are determined by both, loss probability and spatial $\alpha$-particle source distribution which is shown in Fig. 8 by distribution over the flux surface label $s$ of the fusion power in $\alpha$-particles computed for $50 \%$ d-t mixture as

$$
\frac{\mathrm{d} P_{\alpha}}{\mathrm{d} s}=2 \pi n_{d} n_{t}\langle\sigma v\rangle_{d-t} \mathcal{E}_{0} \int_{-\pi}^{\pi} \mathrm{d} \vartheta \sqrt{g} .
$$


Here, $\sqrt{g}$ is metric determinant of coordinates $(s, \vartheta, \varphi), n_{d}, n_{t},\langle\sigma v\rangle_{d-t}$ and $\mathcal{E}_{0}$ are deuterium and tritium densities, transport cross section of d-t reaction (see, e.g., Ref. 21) and initial $\alpha$-particle energy, respectively. Profiles of ion density and temperature entering (25) are the same as in the previous computations of this paper (see Fig. 2). The total power loss is given as

$$
P_{\text {loss }}=\sum_{\text {classes }} \int_{0}^{1} \mathrm{~d} s \frac{\mathrm{d} P_{\alpha}}{\mathrm{d} s} \bar{p}_{L, \text { class }}^{(e)} .
$$

The quantity $\bar{p}_{L, \text { class }}^{(e)}$ for passing particles is given by

$$
\bar{p}_{L, \text { pass }}^{(e)}=\frac{1}{4}\left(\int_{-\pi}^{\pi} \mathrm{d} \vartheta \sqrt{g}\right)^{-1} \sum_{\sigma} \int_{0}^{1} \mathrm{~d} \eta p_{L}^{(e)} \int_{-\pi}^{\pi} \mathrm{d} \vartheta \sqrt{g} \frac{B}{B_{\max }^{(\mathrm{sep})}}\left(1-\eta \frac{B}{B_{\max }^{(\mathrm{sep})}}\right)^{-1 / 2} .
$$

were the summation is over velocity $\operatorname{sign} \sigma$, for trapped particles by

$$
\bar{p}_{L, t r a p}^{(e)}=\left\langle p_{L}^{(e)}\right\rangle_{t r}\left(\int_{-\pi}^{\pi} \mathrm{d} \vartheta \sqrt{g}\right)^{-1} \int_{-\pi}^{\pi} \mathrm{d} \vartheta \sqrt{g}\left(1-\eta \frac{B}{B_{\max }^{(\mathrm{sep})}}\right)^{1 / 2}
$$

and for transient particles by Eq. (27) with replacement of integration interval over $\eta$ by the corresponding domain $\left[1, B_{\max }^{(\mathrm{sep})} / B_{\max }^{(\mathrm{brt})}\right]$. Transient particles constitute a rather small group at the edge. No computations have been performed for this group, but for an upper estimate of their losses, the quantity $p_{L}^{(e)}$ has been extrapolated from the passing domain (dependence of this quantity on $\eta$ is rather weak). The average probabilities $\bar{p}_{L, \text { class }}^{(e)}$ are shown in Fig. 13. Note the strong reduction by shielding of the passing particle contribution which is the main loss channel in vacuum. Finally, the results of integration of different contributions to the power loss in (26), both the absolute values and the values normalized by the total fusion power in $\alpha$-particles which is equal to $P_{\alpha}=91 \mathrm{MW}$ are summarized in Table. I.

\section{SUMMARY AND DISCUSSION}

In this study, $\alpha$-particle losses caused by ELM mitigation coil system in ITER have been estimated for the vacuum RMP model and for the model taking RMP shielding by the plasma into account. As one could expect, shielding of RMPs strongly reduces the width of the region at the outer side of the plasma volume occupied by ergodic passing $\alpha$-particle orbits. This reduction, however, is smaller than such a reduction for magnetic field lines 
and it is different for co- and counter-passing orbits. Nevertheless, it is sufficient to prevent noticeable losses predicted by the vacuum RMP model. Indeed, as follows from Table I, the main channel of losses in the vacuum approximation for the perturbation field is the loss of passing particles which cause $4.7 \%$ loss of $\alpha$-particle fusion power. Together with the trapped particle contribution, $\alpha$-particle fusion power loss exceeds $5 \%$ with particles being lost without significant slowing down by electrons. If the shielding of RMPs is taken into account, the passing particle channel of losses is practically eliminated.

It should be noted that external magnetic field perturbations can also be amplified by the plasma. E.g., this happens if perturbation spectrum couples to marginally stable perturbations which produce kinklike distortions of the plasma ${ }^{22}$. In particular, an amplification of the perturbation field at the plasma edge has been suggested as a most probable explanation for the increased (compared to the vacuum perturbation field) divertor strike point splitting observed in DIII-D ${ }^{23}$. At the same time, amplification or even sustainment at the vacuum level of the RMPs in the core plasma is evidently not the case in the regimes of practical interest, because such RMPs would significantly degrade the parameters of the core plasma making the question of $\alpha$-particle confinement a secondary issue.

\section{Acknowledgments}

This work, supported in part by the European Commission under the contract of Associations between EURATOM, Austrian Academy of Sciences and IPP Greifswald was carried out within the framework of the European Fusion Development Agreement. The views and opinions expressed herein do not necessarily reflect those of the European Commission or those of the ITER Organization. Additional funding is provided by the Austrian Science Foundation, FWF, under contract number P19889-N16.

1 T. E. Evans, R. A. Moyer, P. R. Thomas, J. G. Watkins, T. H. Osborne, J. A. Boedo, E. J. Doyle, and M. E. Fenstermacher, Phys. Rev. Letters 92, 235003 (2004).

${ }^{2}$ Y. Liang, H. R. Koslowski, P. R. Thomas, E. Nardon, B. Alper, P. Andrew, Y. Andrew, G. Arnoux, Y. Baranov, M. Bécoulet, et al., Phys. Rev. Lett. 98, 265004 (2007).

3 M. Becoulet, E. Nardon, G. Huysmans, W. Zwingmann, P. Thomas, M. Lipa, R. Moyer, 
T. Evans, V. Chuyanov, Y. Gribov, et al., Nucl. Fusion 48, 024003 (2008).

4 I. Joseph, T. Evans, A. Runov, M. Fenstermacher, M. Groth, S. Kasilov, C. Lasnier, R. Moyer, G. Porter, M. Schaffer, et al., Nucl. Fusion 48, 045009 (2008).

5 M. F. Heyn, I. B. Ivanov, S. V. Kasilov, W. Kernbichler, I. Joseph, R. A. Moyer, and A. M. Runov, Nuclear Fusion 48, 024005 (2008).

6 A. Cole and R. Fitzpatrick, Phys. Plasmas 13, 032503 (2006).

7 M. Schaffer et al. (2010), private communication.

8 A. Loarte et al., in Fusion Energy 2010 (Proc. 23rd Int. Conf. Daejeon, 2010) (IAEA, Vienna, 2010), CD-ROM file ITR 1-4. http://wwwpub.iaea.org/mtcd/meetings/PDFplus/2010/cn180/cn180_papers/itr_1-4.pdf.

9 T. Casper et al., in Fusion Energy 2010 (Proc. 23rd Int. Conf. Daejeon, 2010) (IAEA, Vienna, 2010), CD-ROM file ITR/P1-19, accepted Nucl. Fusion http://wwwnaweb.iaea.org/napc/physics/FEC/FEC2010/html/index.htm.

10 M. F. Heyn, I. B. Ivanov, S. V. Kasilov, and W. Kernbichler, Nuclear Fusion 46, S159 (2006).

11 I. B. Ivanov, M. F. Heyn, S. V. Kasilov, and W. Kernbichler, Phys. Plasmas 18, 022501 (2011).

12 E. Nardon, P. Tamain, M. Bécoulet, G. Huysmans, and F. L. Waelbroeck, Nucl. Fusion 50, $034002(2010)$.

13 N. G. van Kampen, Stochastic processes in physics and chemistry (Elsevier Science Publishers B.V, Amsterdam, 1992).

14 A. I. Morozov and L. S. Solov'ev, in Reviews of Plasma Physics, edited by M. A. Leontovich (Consultants Bureau, New York, 1966), vol. 2.

15 S. S. Abdullaev, A. Wingen, and K. H. Spatschek, Phys. Plasmas 13, 042509 (2006).

16 W. Lotz, P. Merkel, J. Nuehrenberg, and E. Strumberger, Plasma Phys. Contr. Fus. 34, 1037 (1992).

17 M. de Rover, N. J. Lopes Cardozo, and A. Montvai, Phys. Plasmas 3, 4468 (1996).

18 A. M. Runov, D. Reiter, S. V. Kasilov, W. Kernbichler, and M. F. Heyn, Phys. Plasmas 8, 916 (2001).

19 S. V. Kasilov, W. Kernbichler, V. V. Nemov, and M. F. Heyn, Phys. Plasmas 9, 3508 (2002).

20 R. J. Goldston, R. B. White, and A. H. Boozer, Phys. Rev. Lett. 47, 647 (1981).

21 T. J. Dolan, Fusion Research: Principles, experiments and technology (Pergamon Press, New York, 1982). 
22 A. H. Boozer, Phys. Rev. Lett 86, 5059 (2001).

23 M. W. Jakubowski, T. E. Evans, M. E. Fenstermacher, M. Groth, C. J. Lasnier, A. W. Leonard, O. Schmitz, J. W. Watkins, T. Eich, W. Fundamenski, et al., Nuclear Fusion 49, 095013 (2009). 
Figures

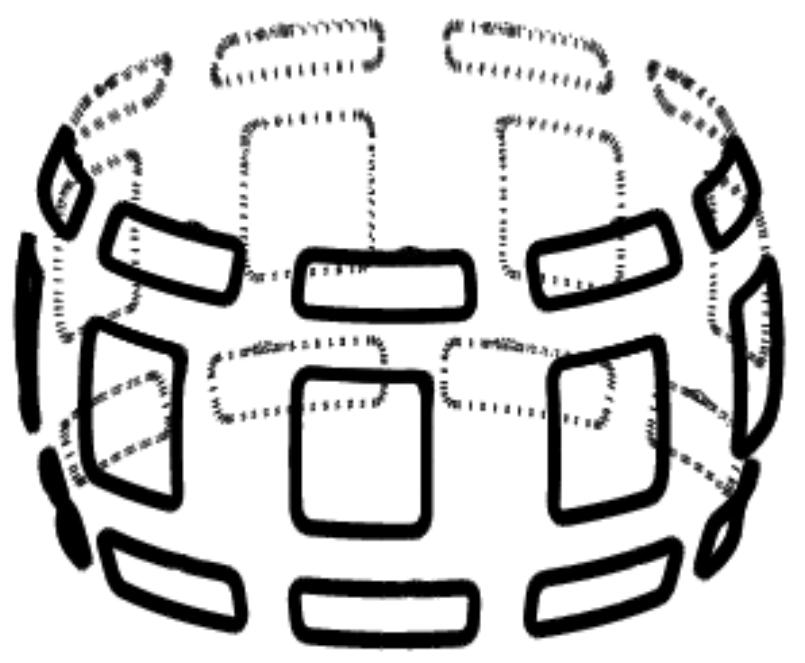

FIG. 1: Perturbation coil geometry.

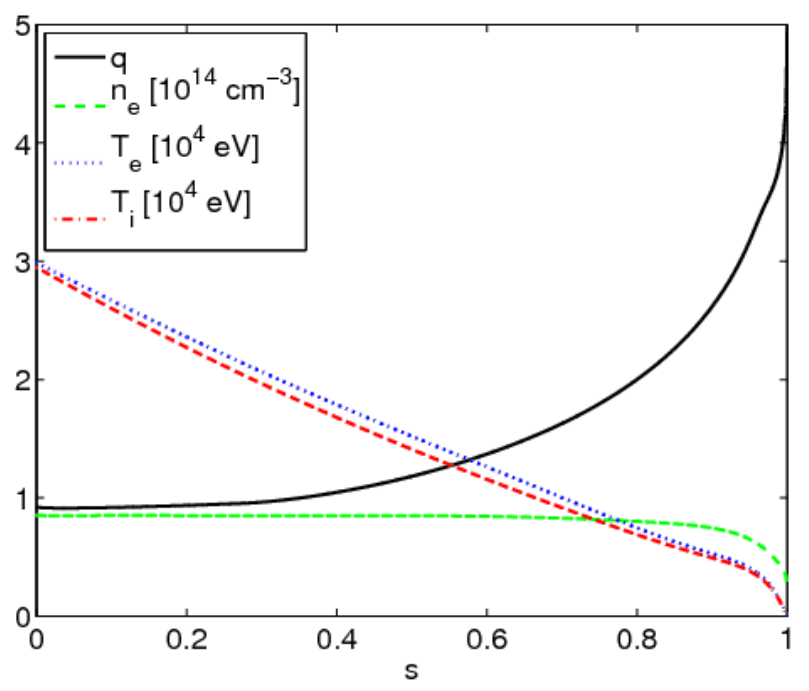

FIG. 2: Equilibrium plasma parameter profiles. 

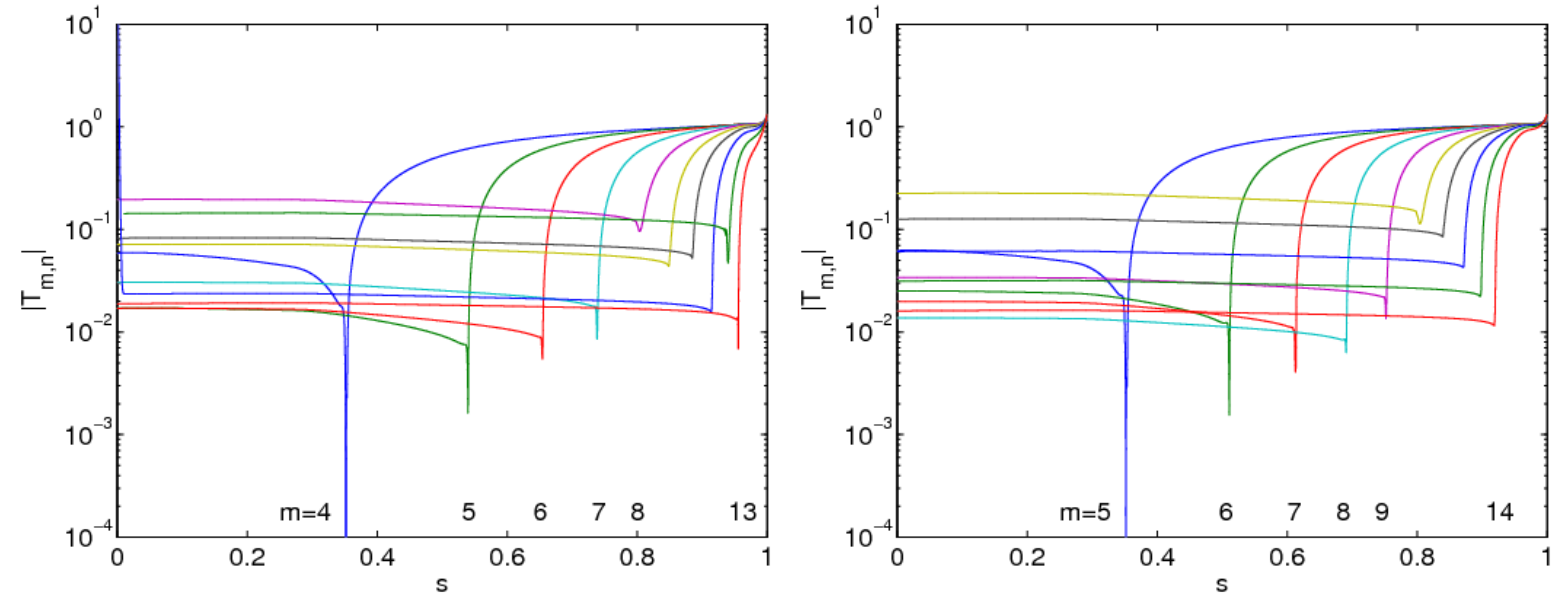

FIG. 3: Formfactors $\left|T_{m, n}\right|$ vs the normalized poloidal flux $s$ for $n=-4$ (left) and $n=-5$ (right). Poloidal wave numbers $m$ label respective curves.
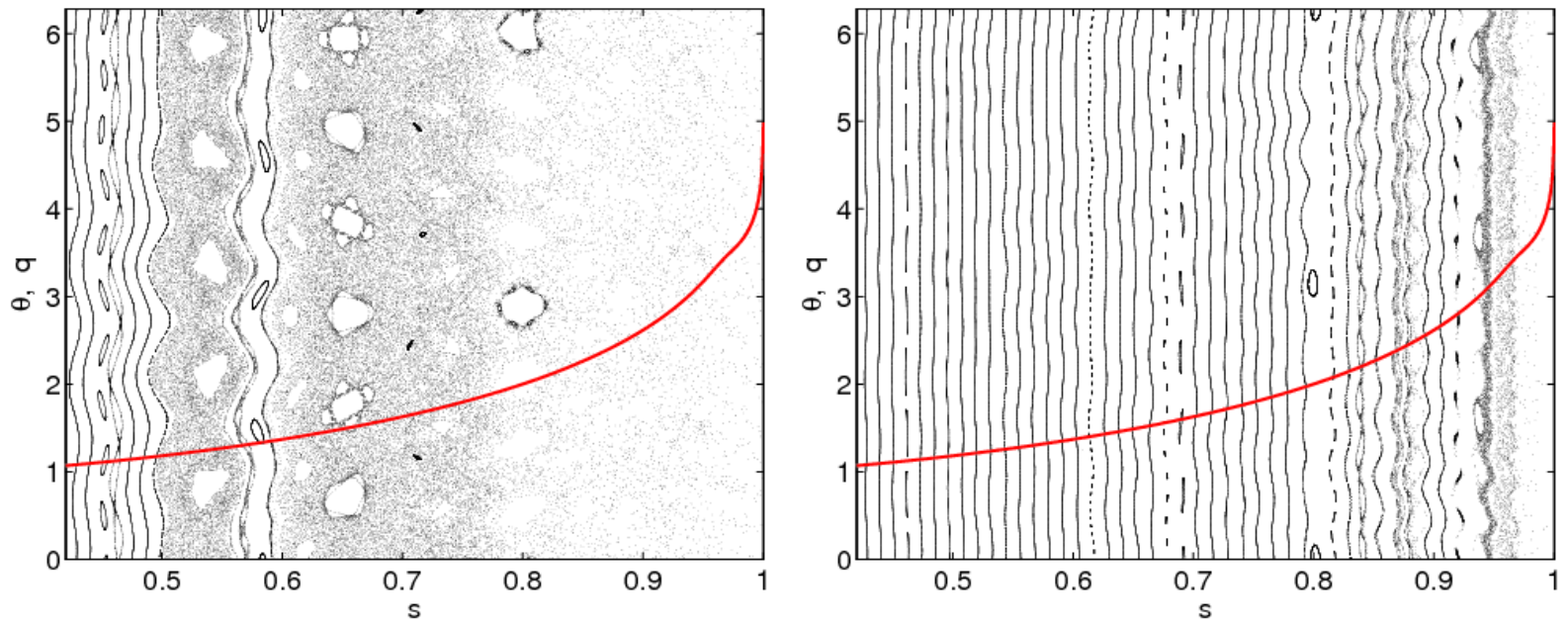

FIG. 4: Poincaré plots of the magnetic field for vacuum RMP model (left) and with the account of RMP shielding by the plasma (right). Red line shows the safety factor $q(s)$. 

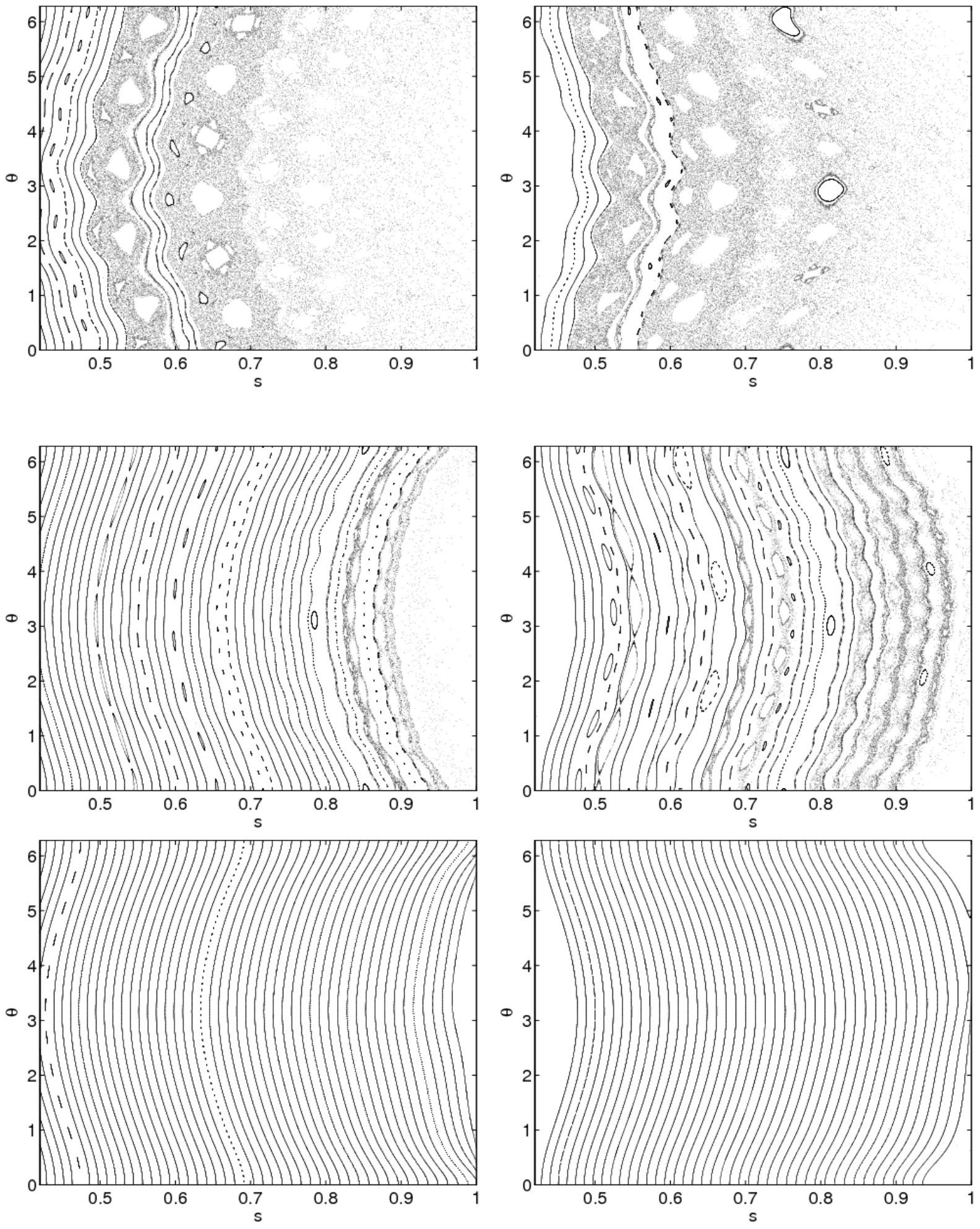

FIG. 5: Poincaré plots of co-passing (left panel) and counter-passing (right panel) $\alpha$-particle orbits for vacuum RMP model (top), with the account of RMP shielding by the plasma (middle) and in absence of RMPs (bottom). 


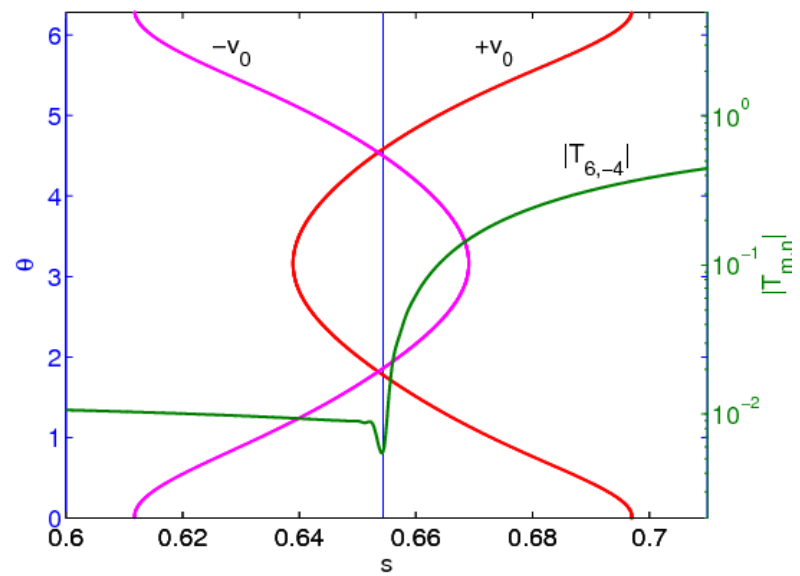

FIG. 6: Unperturbed resonant orbits for co-passing $\left(v_{0}\right.$, red) and counter-passing $\left(-v_{0}\right.$, magenta) $\alpha$-particles and the unperturbed resonant flux surface (blue) for $n=-4, m=6$ resonance. Green curve shows the respective formfactor $\left|T_{6,-4}\right|$. 

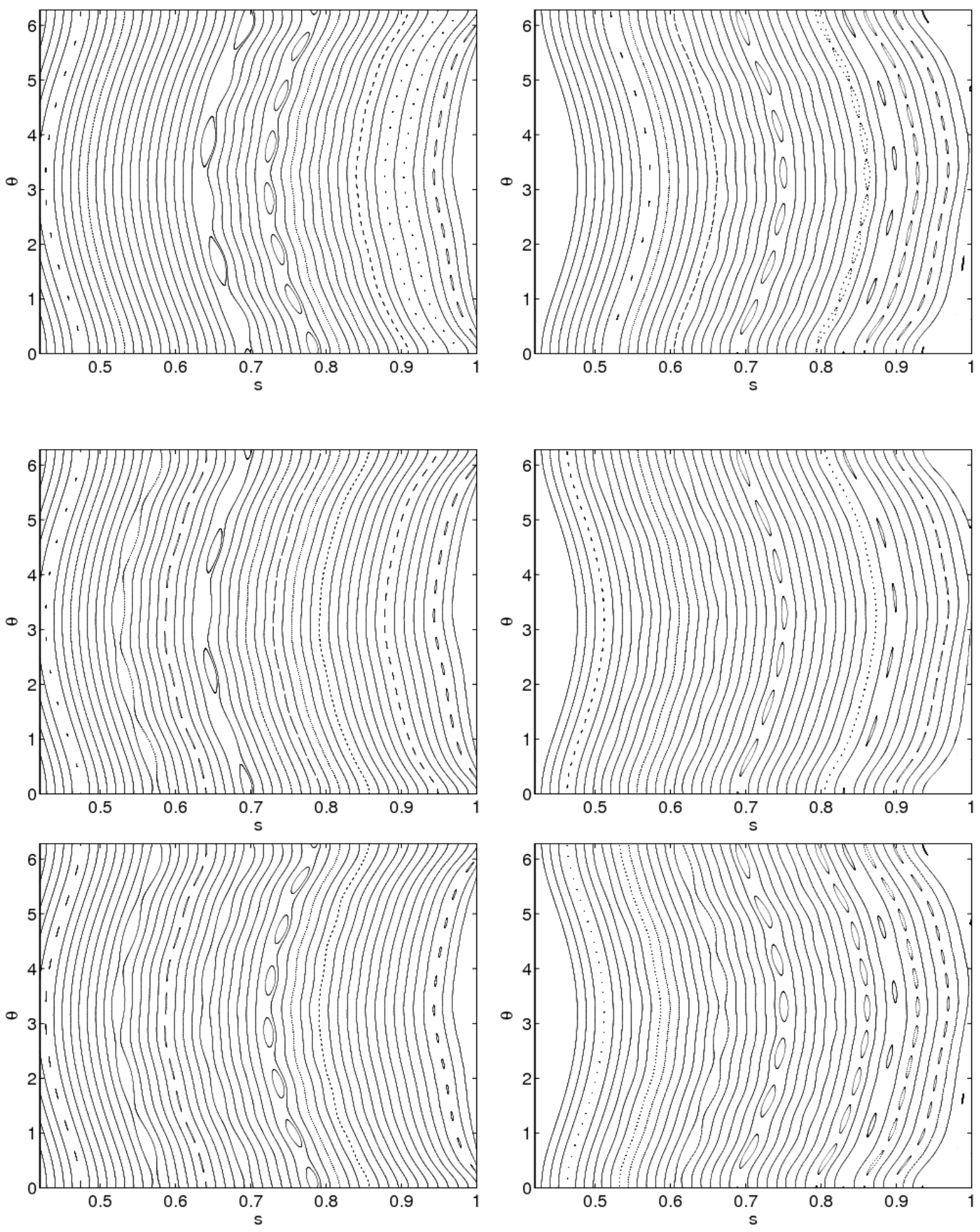

FIG. 7: Poincaré plots of co-passing (left panel) and counter-passing (right panel) $\alpha$-particle orbits for a shielded $n=-4, m=6$ RMP harmonic (top), $n=-4, m=5$ harmonic (middle) and superposition of these harmonics (bottom). 


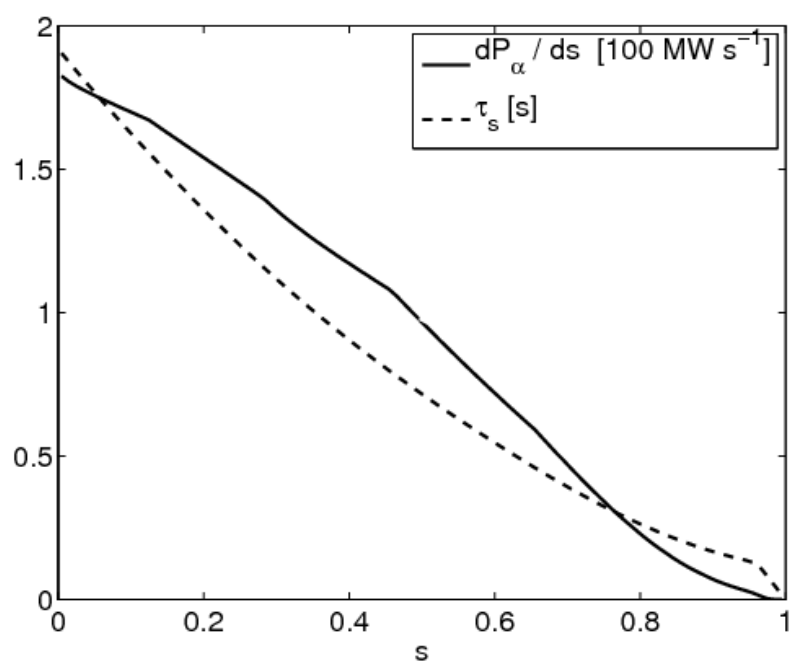

FIG. 8: $\alpha$-particle fusion power distribution over normalized poloidal flux and $\alpha$-particle slowing down time by electrons for the parameters of Fig. 2 .
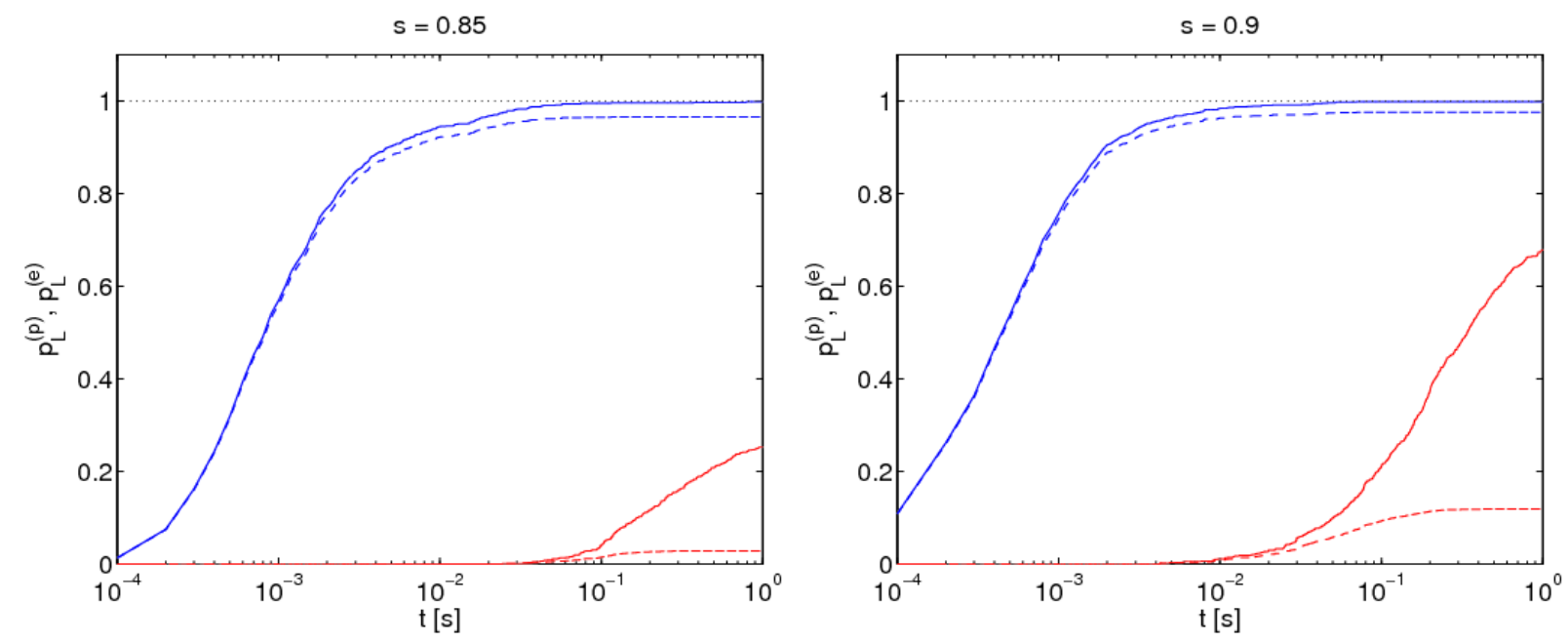

FIG. 9: Particle $p_{L}^{(p)}$ (solid) and energy $p_{L}^{(e)}$ (dashed) loss probabilities for strongly counter-passing particles $(\eta=0)$ as functions time after particle birth for particles born at $s=0.85$ flux surface (left) and $s=0.9$ flux surface (right). Blue - vacuum perturbation model, red - shielded perturbation model. 

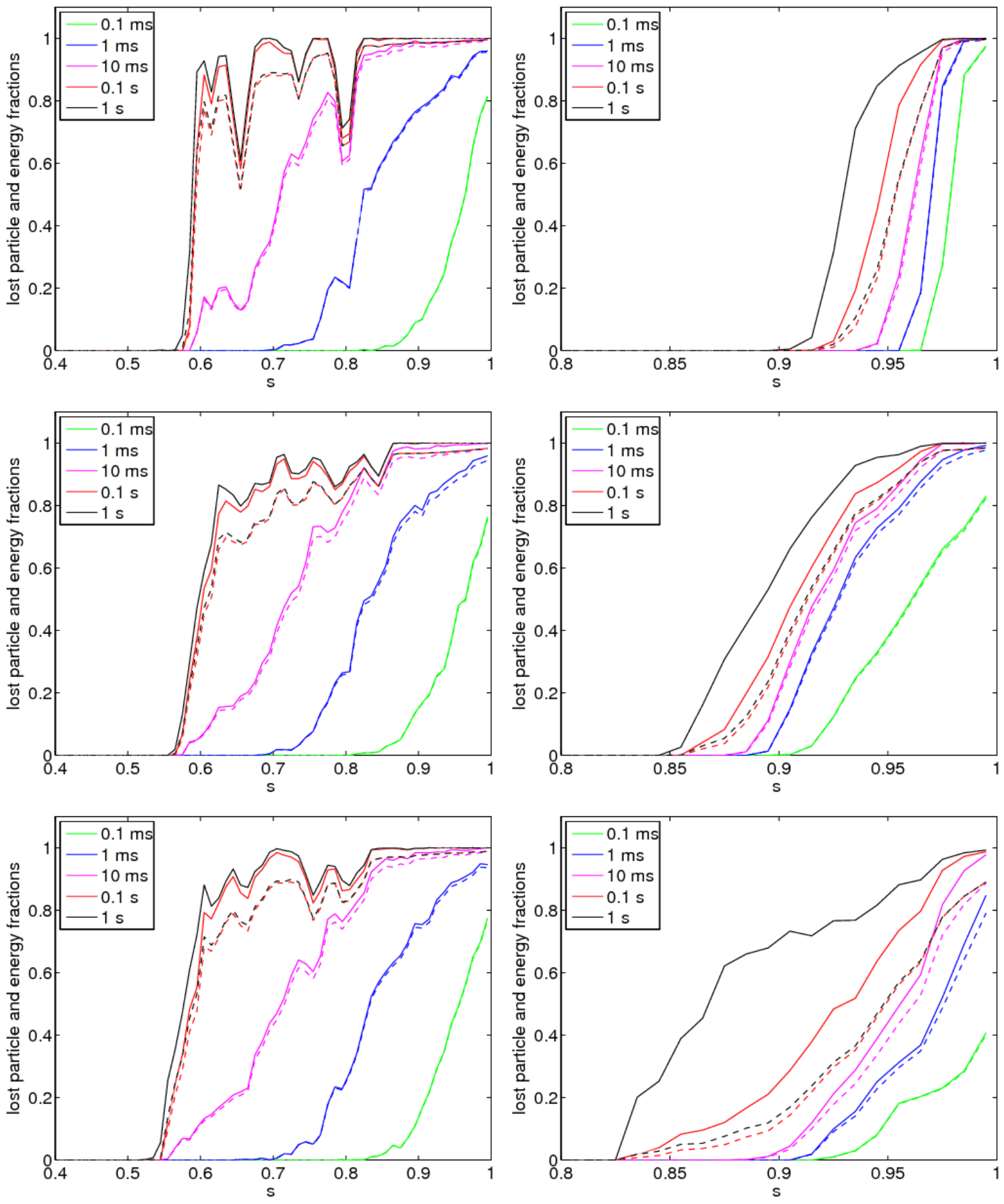

FIG. 10: Dynamics of strongly passing $(\eta=0)$ particle $p_{L}^{(p)}$ (solid) loss probability and energy $p_{L}^{(e)}$ (dashed) loss probability for vacuum perturbation (left) and for shielded perturbation (right). Upper panel - field lines, middle panel - co-passing, lower panel - counter-passing. Note the difference in normalized poloidal flux, $s$, range between vacuum and plasma cases. 


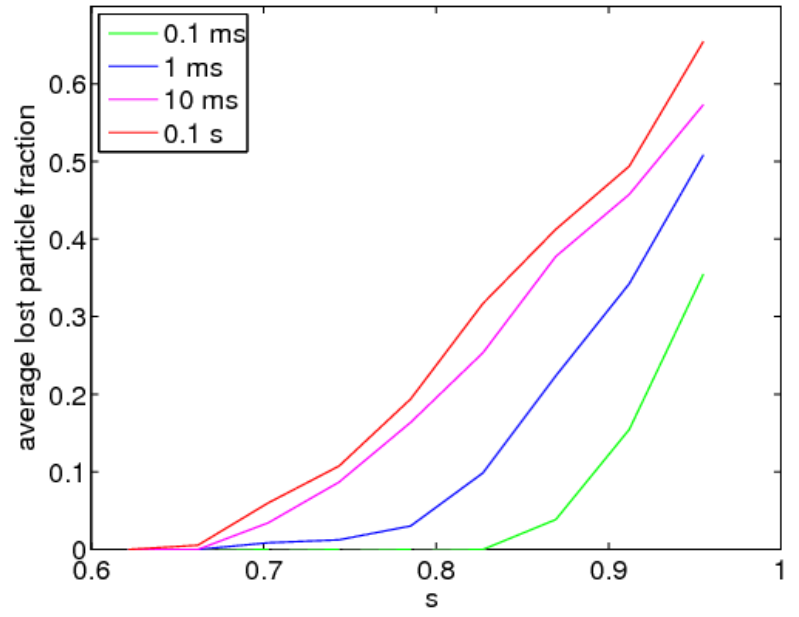

FIG. 11: Dynamics of trapped particle loss probability $\left\langle p_{L}^{(p)}\right\rangle_{t r}$ for vacuum perturbation field.

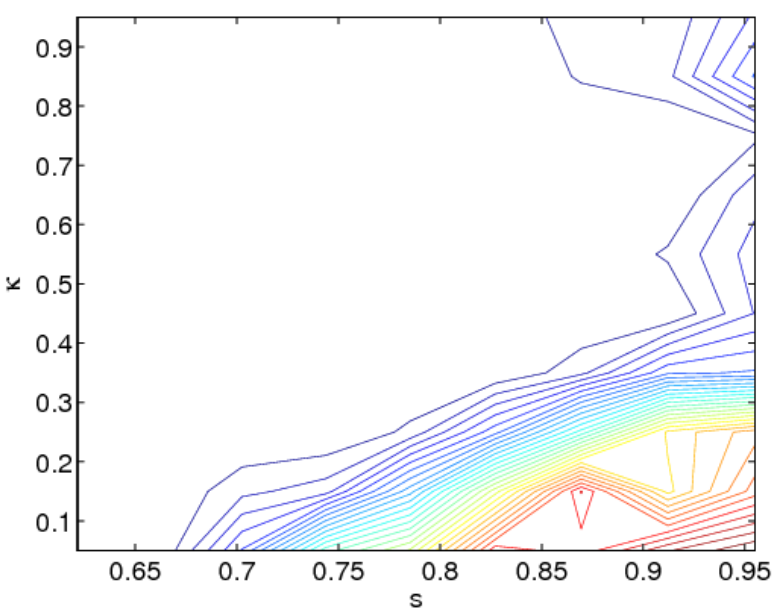

FIG. 12: Distribution of trapped particle loss probability $p_{L}^{(p)}$ for vacuum perturbation field. 


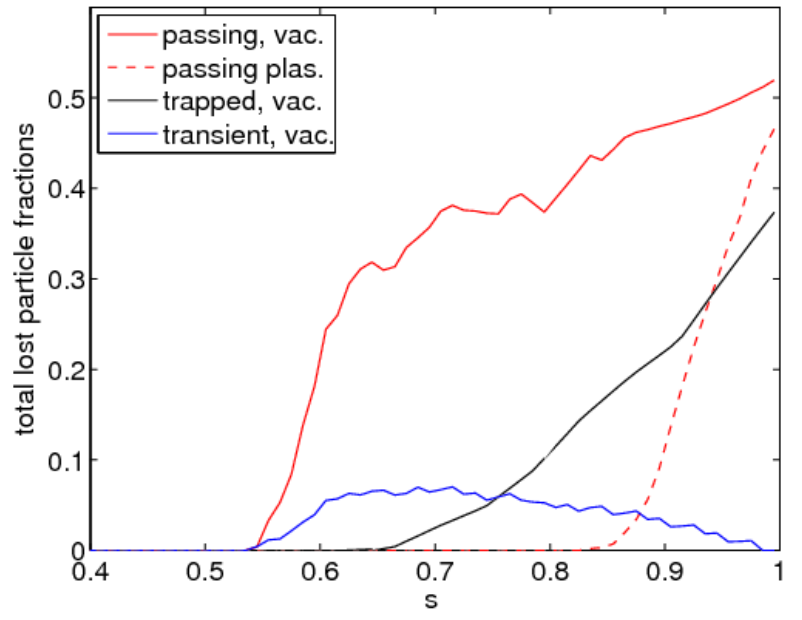

FIG. 13: Flux surface and momentum space averaged loss probabilities $\bar{p}_{L, c l a s s}^{(e)}$ for various particle classes in vacuum and for passing particles in plasma. For transient particles the upper estimate is plotted (vacuum case). 


\section{Tables}

TABLE I: Energy losses.

\begin{tabular}{|c|c|c|c|c|}
\hline & passing, vac. & passing, plas. & trapped, vac & transient, vac. \\
\hline MW & 4.25 & 0.08 & 0.51 & 0.74 \\
\hline$\%$ & 4.66 & 0.09 & 0.56 & 0.82 \\
\hline
\end{tabular}

